\title{
Medievalista
}

Online

$28 \mid 2020$

Número 28

dois insignes medievistas franceses que o COVID 19 vitimou

\section{In memoriam Francisc Rapp (n. 1926) e Michel} Parisse (n. 1936)

dois insignes medievistas franceses que o COVID 19 vitimou

In memoriam Francisc Rapp (b. 1926) and Michel Parisse (b.1936): two

distinguished medievalists victims of COVID 19

\section{Armando Luís de Carvalho Homem}

\section{(2) OpenEdition}

\section{Journals}

Edição electrónica

URL: http://journals.openedition.org/medievalista/3277

DOI: $10.4000 /$ medievalista.3277

ISSN: 1646-740X

Editora

Instituto de Estudos Medievais - FCSH-UNL

Edição impressa

Paginação: 11-20

Refêrencia eletrónica

Armando Luís de Carvalho Homem, «In memoriam Francisc Rapp (n. 1926) e Michel Parisse (n. 1936)», Medievalista [Online], 28 | 2020, posto online no dia 01 julho 2020, consultado o 23 março 2021. URL: http://journals.openedition.org/medievalista/3277 ; DOI: https://doi.org/10.4000/medievalista.3277

Este documento foi criado de forma automática no dia 23 março 2021.

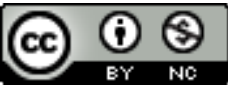

Mediavalista está licenciado com uma Licença Creative Commons - Atribuição-NãoComercial 4.0 Internacional. 
dois insignes medievistas franceses que o COVID 19 vitimou

\section{In memoriam Francisc Rapp (n. 1926) e Michel Parisse (n. 1936)}

dois insignes medievistas franceses que o COVID 19 vitimou

In memoriam Francisc Rapp (b. 1926) and Michel Parisse (b.1936): two

distinguished medievalists victims of COVID 19

Armando Luís de Carvalho Homem

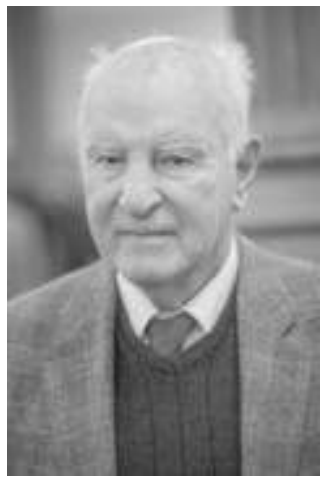

Francis Rapp 


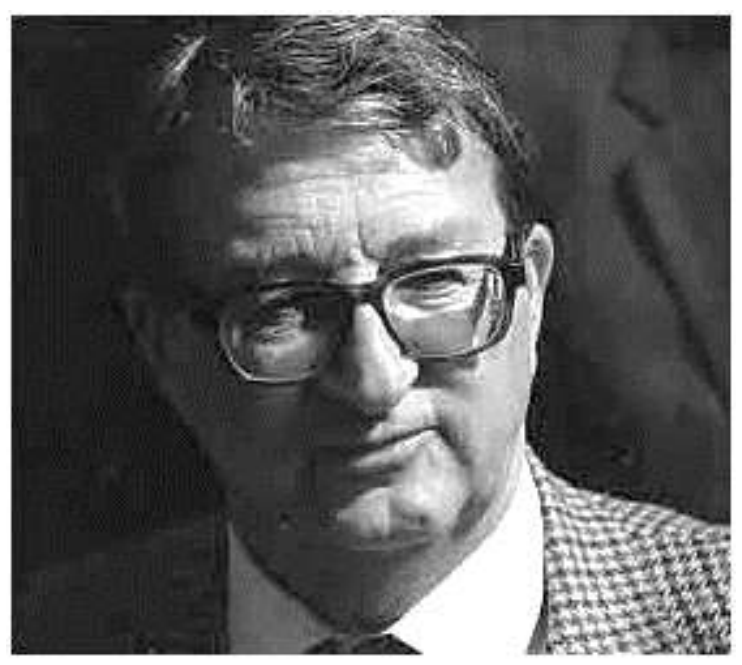

Michel Parisse

1 A pandemia em que estamos mergulhados não tem poupado homens e mulheres de Ciência, artistas, criadores literários, músicos, actores ...: pensemos, tão somente e entre nós, na imunologista Maria de Sousa (1939-2020).

2 Março e Abril do ano em curso levaram-nos dois nomes sonantes do medievismo francês. Evoquemo-los, portanto.

3 Membro do Institut de France (Académie des Inscriptions et Belles-Lettres) [1993 ss.], professor emérito da Universidade de Estrasburgo, Francis Rapp nasceu nesta cidade a 27 de Junho de 1926. Feitos os estudos secundários e superiores em colégios e liceus e por último na Universidade da sua cidade-natal; entusiasta, também, do escotismo ${ }^{1}$, em 1952 obterá a agrégation d'Histoire, com elevada classificação.

4 Seguiram-se alguns tempos como bolseiro da Fondation Dosne-Thiers (1956-1961). No último ano mencionado torna-se chargé de cours da Universidade de Nancy e em 1972 assistente de História Medieval da Université Marc Bloch de Estrasburgo ${ }^{2}$. No mesmo ano obtém o doctorat d'État, e em 1974 ascende a maître de conférences e depois a professor em Estrasburgo.

5 A tese principal para o doctorat intitulou-se Réformes et réformation à Strasbourg. Église et socièté dans le diocese de Strasbourg (1450-1525) ${ }^{3}$. Teve como orientador Robert Folz (1910-1996) $)^{4}$ e como co-orientador Jean Schneider (1903-2004)5. Ou seja, Rapp surge-nos geograficamente marcado por cidades, figuras e instituições da Alsácia-Lorena, bem como pelos destinos iniciais dos Annales. Para além disto, a sua geografia predominante tê-lo-á levado a um encarar em termos colaborantes do catolicismo e do protestantismo.

6 A sua cátedra de Estrasburgo não o impediu de ensinar também "História do Cristianismo" na Faculdade de Teologia Protestante da mesma cidade (1972-1991), e ainda na Universidade de Neuchâtel e em múltiplas escolas superiores da Europa e da América.

7 Em 1971 editou, na colecção "Nouvelle Clio", o volume sobre Igreja e vida religiosa nos finais da Idade Média ${ }^{6}$, que de algum modo faz conjunto com os volumes sincrónicos de Jacques Heers (1924-2013) - para a história económica e social - e de Bernard Guenée (1927-2010) - para as estruturas políticas?. 
E em 1989 dar-nos-ia Les origines médiévales de l'Allemagne moderne ${ }^{8}$. As sínteses, aliás, nunca deixaram de o atrair, sobre o Império germânico, Estrasburgo (a cidade, religião e reformas religiosas, a diocese...), a Alsácia, instituições monásticas alemãs ${ }^{9}$, entre outros.

Morreu a 29 de Março do ano em curso, no Centre hospitalier universitaire d'Angers, onde se encontrava internado.

Foi casado com Marie-Rose Sutter (1936-2018), e de tal matrimónio houve três filhos.

11 Michel Parisse nasceu em 1936 em Void-Vacon (Mosa). Fez os seus estudos liceais e superiores em Nancy, e em 1959 obteve a agrégation d'histoire. Professor liceal em Metz de 1959 a 1965, neste último ano começa a ensinar, primeiro como assistente, depois como professor de História Medieval, na Universidade de Nancy-II ${ }^{10}$. Obtido, entretanto, o doctorat de III cycle (1966, tese: Actes des évêques de Metz [1120-1179]) e o doctorat d'État (1975, tese: La Noblesse lorraine [XI ${ }^{e}$ XIII ${ }^{e}$ siècle]), torna-se definitivamente professor em Nancy-II, instituição a que ficará ligado até 1992.

Será entretanto Director do ARTEM (Atelier de recherche sur les textes médiévaux et leur traitement assisté), de 1983 à 1993. O ARTEM, fundado em 1966 enquanto "Centre de Recherches et d'Applications Linguistiques", pela iniciativa de Jean Schneider de desenvolver a pesquisa sobre os textos, graças aos recém-nascidos utensílios da ciência informática, assume à partida como tarefa o estudo do vocabulário e da língua dos textos diplomatísticos da Idade Média, constituindo um recenseamento exaustivo dos actos originais conservados em França até 1120 (neste eixo de trabalho, a sucessão de Michel Parisse foi assegurada por Benoît-Michel Tock). Outros eixos ou "ateliers" vieram completar o primeiro (atelier Vincent de Beauvais em 1974, atelier de textos monásticos em 1986 e atelier de prosopografia e de história social em 1994). Enquanto director do ARTEM, Michel Parisse foi substituído por Pierre Pégeot.

Dirigiu igualmente a Mission historique française na Alemanha, em Göttingen, de 1985 a 1991. E em 1991-1992 estava de regresso a Nancy.

Em 1993 foi eleito professor de História Medieval em Paris-I, e aí ficará até se aposentar (2002).

15 Pela sua acção à frente do ARTEM e depois na Universidade de Paris-I, nomeadamente no seio do Laboratoire de médiévistique occidentale de Paris (LaMOP), contribuiu para o desenvolvimento dos estudos medio-latinos (introduziu o estudo do latim medieval nos cursos de licenciatura e de post-graduação de Paris-I) e estudos de diplomática e de paleografia, particularmente no que toca os actos episcopais, as pancartas e os cartulários.

Num balanço de carreira, dir-se-á que os seus campos de investigação foram a Lorena medieval (incluindo a nobreza respectiva), o Sacro-Império, os cónegos regulares e as religiosas, o latim e as fontes diplomatísticas. Embora as notícias biográficas a que tive acesso não indiquem o(s) orientador(es) de tese, pude, entretanto, verificar a muita consideração que suscitava junto de figuras como Robert-Henri Bautier (1922-2010) e Robert Fossier (1927-2012).

Lisboa, 3 de Maio de 2020 


\section{BIBLIOGRAFIA}

Francis Rapp: Bibliografia activa (selecção)

Inventaire des sources manuscrites de l'histoire d'Alsace conservées dans les bibliothèques publiques de France. Paris: Fédération des sociétés d'histoire et d'archéologie d'Alsace, 1956.

Le Château-Fort dans la vie médiévale: Le Château-Fort et la Politique territoriale. Strasbourg: Centre d'Archéologie médiévale, 1968.

L'Église et la Vie Religieuse en Occident à la fin du Moyen Âge. Paris: PUF, 1971 [coll. "Nouvelle Clio", n.․ㄹ․

Réformes et Réformation à Strasbourg. Église et Société dans le diocèse de Strasbourg (1450-1525). Paris: Ophrys, 1974.

(Dir.) Grandes Figures de l'humanisme alsacien. Courants, milieux, destins. Strasbourg: Istra, 1978.

(Dir.) Histoire de Strasbourg des origines à nos jours. 9 vols. Strasbourg: Dernières nouvelles de Strasbourg, 1981.

Les Origines médiévales de l'Allemagne moderne. De Charles IV à Charles Quint (1346-1519). Paris: Aubier, 1989.

Histoire des diocèses de France: Le Diocèse de Strasbourg. Paris: Éditions Beauchesne, 1997.

(Colaboration avec. Claude Muller) Koenigsbruck : l'histoire d'une abbaye cistercienne. Strasbourg: Société d'histoire et d'archéologie du Ried Nord, 1998.

Le Saint-Empire romain germanique, d'Otton le Grand à Charles Quint. Paris: Éditions du Seuil, 2003.

(Dir.) Christentum und Kirche im 4. und 5. Jahrhundert. Heidelberga: Universitätsverlag Winter, 2003

(Dir.) Christentum IV: Zwischen Mittelalter und Neuzeit (1378-1552). Stugard: Kohlhammer, 2006.

Maximilien d'Autriche. Paris: Éditions Tallandier, 2007.

(Dir.) Protestants et Protestantisme en Alsace de 1517 à nos jours. Strasbourg: Fédération des sociétés d'histoire et d'archéologie d'Alsace, 2007.

(Dir.) Strasbourg. Paris: La Nuée Bleue, 2010.

Francis Rapp: Bibliografia passiva

BISCHOFF, Georges - “Francis Rapp”. Arche. Arts, Civilisation et histoire de l'Europe (2020/03/20) [em linha]. [Consultado a 2 Maio 2020]. Disponível em https://arche.unistra.fr/actualites-agenda/filinfos/actualite/?tx_ttnews\%5Btt_news\%5D=11535\&cHash=5cfb23a51f7fe7647d6d21be42cd1a85.

CATINCHI, Philippe-Jean - "Le Médiéviste Francis Rapp est mort". Le Monde [em linha] (2020/03/30). [Consultado a 2 Maio 2020]. Disponível em https://www.lemonde.fr/disparitions/ article/2020/03/30/le-medieviste-francis-rapp-est-mort_6034926_3382.html.

"Francis Rapp". Académie d'Alsace des Sciences, Lettres et Arts [em linha]. [Consultado a 3 Maio 2020]. Disponível em http://www.academie-alsace.fr/comit\%C3\%A9/comit\%C3\%A9-d-honneur/ francis-rapp/.

FUCHS, François-Joseph - "Francis Rapp”. in Nouveau Dictionnaire de Biographie alsacienne. Fasc. 30. Strasbourg: Fédération des Sociétés d'Histoire et d'Archéologie d'Alsace, 1997, pp. 3083-3084. 
“L'Historien Francis Rapp meurt à 93 ans du coronavírus", Le Figaro [em linha] (2020/03/30). [Consultado a 2 Maio 2020]. Disponível em https://www.lefigaro.fr/culture/l-historien-francisrapp-meurt-a-93-ans-du-coronavirus-20200330.

MEHL, Jean-Michel - "Francis Rapp: un historien amoureux". Mélanges offerts à Francis Rapp, Revue d'Alsace 122 (1996), pp. 5-8.

RACINE, Pierre - “Hommage à Francis Rapp”. Revue des Sciences religieuses 69-2 (1995), pp. 143-145.

"Rapp Francis, Jean, Josep". in Académie des Inscriptions et Belles-Lettres [em linha]. [Consultado a 2 Maio 2020]. Disponível em https://www.aibl.fr/membres/academiciens-depuis-1663/article/ rapp-francis-jean-joseph?lang=fr.

THEIS, Laurent - "Francis Rapp est mort", L'histoire [em linha] (2020/04/02). [Consultado a 2 Maio 2020]. Disponível em https://www.lhistoire.fr/hommage/francis-rapp-est-mort.

Michel Parisse: Bibliografia activa (selecção)

“Le nécrologe de Gorze. Contribution à l'histoire monastique". Mémoires des Annales de l'Est 40 (1971).

Actes des princes lorrains. Nancy: Université de Nancy-II, U.E.R. de recherche régionale, 1972-1974.

"Les chartes des évêques de Metz au XII siècle: étude diplomatique et paléographique". Archiv für Diplomatik 22 (1976), pp. 272-316.

Histoire de Nancy. Toulouse: Privat, 1978.

La Lorraine monastique au Moyen Âge. Nancy: Service des publications de l'Université de Nancy II, 1981.

"Les Benedictines de Lorraine et leurs Documents Necrologiques". Consuetudines monasticae: eine Festgabe für Kassius Hallinger aus Anlass seines 70. Geburtstages (Rome: Pontificio Atenos S. Anselmo) 85 (1982), pp. 249-262.

La Noblesse lorraine (XI ${ }^{e}-\mathrm{XIII}^{e}$ siècle). Ed. parcial: Noblesse et chevalerie en Lorraine médiévale: les familles nobles du XI ${ }^{e}$ au XIII e siècle. Nancy: Service des publications de l'Université de Nancy II, 1982.

Les Nonnes au Moyen Âge. Lepuy-en-Velay: C. Benneton, 1983.

La tapisserie de Bayeux: Un documentaire du XI' siècle. S.l.: Denoël, 1983.

Les religieuses en France au XIII ${ }^{e}$ siècle. Nancy: Service des publications de l'Université de Nancy II, 1985 (2 éd., 1989).

Histoire de la Lorraine. Toulouse: Privat, 1987.

Austrasie, Lotharingie, Lorraine. Metz: Editions Serpenoise; Nancy: Presses universitaires de Nancy, 1990.

Actes des évêques de France. Nancy: Presses universitaires de Nancy, 1991.

Atlas de la France de l'an Mil: état de nos connaissances. Paris: Picard, 1994.

Atlas de l'an Mil. Paris: Picard, 1994.

La Vie de Jean, abbé de Gorze. Paris: Picard, 1999. Apresentação e tradução da obra.

Les Médiévistes français. Paris: Picard, 2001.

Allemagne et Empire au Moyen Âge: 400-1510. Paris: Hachette, 2002. 
Manuel de paléographie médiévale: manuel pour grands commençants. Paris: Picard, 2006.

Allemagne et Empire au Moyen âge. $2^{\mathrm{e}}$ éd. revue et augmentée. Paris: Hachette supérieur, 2008.

Michel Parisse: obras colectivas

(Dir., c/ Stéphane Gaber et Gérard Canini). Grandes dates de l'histoire lorraine. Nancy: Service des publications de l'Université de Nancy-II, 1982.

(Dir., c/ Xavier Barral i Altet). Colloque Hugues Capet, 987-1987, la France de l'an mil. Le roi de France et son royaume autour de l'An mil. Paris: Picard, 1992.

(Dir., c/ Otto Gerhard Oexle). L'abbaye de Gorze au X $X^{e}$ siècle: table ronde de Gorze, septembre 1988. Nancy: Presses universitaires de Nancy, 1993.

(Dir., c/ Olivier Guyotjeannin e Laurent Morelle). Les cartulaires: actes de la Table ronde organisée par l'Ecole nationale des chartes et le GDR 121 du CNRS, Paris, 5-7 décembre 1991. Paris: École des chartes, 1993.

(Dir., c/ Sylvain Gouguenheim, Pierre Monnet e Joseph Morsel). L'Allemagne au XIII siècle: de la Meuse à l'Oder. Paris: Picard, 1994.

(Dir., c/ Pierre Heili). Les chapitres de dames nobles entre France et Empire: actes du colloque d'avril 1996. Remiremont: Société d'histoire locale de Remiremont / Messene, 1998.

(Dir., c/ Monique Bourin). L'Europe au siècle de l'an Mil. Paris: Hachette, 1999.

(Dir., c/ Monique Goullet). Les historiens et le latin médiéval: colloque tenu à la Sorbonne, les 9, 10 et 11 septembre 1999. Paris: Presses de la Sorbonne, 2001.

(Dir., c/ Monique Goullet). Apprendre le latin médiéval: manuel pour grands commençants, $3^{\mathrm{e}}$ éd. Revue et corrigée. Paris: Picard, 2005.

(Dir.) Les chanoines réguliers: émergence et expansion, XIe - XIIIe siècles, Colloque international du Puyen-Velay, 29 juin-1er juillet 2006. Saint-Étienne: Publications de l'Université de Saint-Étienne, 2009.

Michel Parisse: Bibliografia passiva

GAZEAU, Véronique - "Michel Parisse est mort". L'histoire [em linha] (2020/04/06). [Consultado a 2 Maio 2020]. Disponível em https://www.lhistoire.fr/hommage/michel-parisse-est-mort.

MORELLE, Laurent - "Mort du médiéviste Michel Parisse, emporté par le Covid-19 à l'âge de 83 ans". Le Monde [em linha] (2020/04/20). [Consultado a 2 Maio 2020]. Disponível em https:// www.lemonde.fr/disparitions/article/2020/04/20/la-mort-du-medieviste-michelparisse_6037180_3382.html.

PERREAUX, N. - "Décès de Michel Parisse”. Les Carnets du LaMOP [em linha] (2020/04/07).

[Consultado a 3 Maio 2020]. Disponível em https://lamop.hypotheses.org/6738.

\section{NOTAS}

1. O que, a partir de 1942, lhe serviu para evitar a incorporação conscrita nas forças alemãs, ao que era refractário.

2. Entre 1968 e 2008 a Universidade em causa dividiu-se em Strasbourg-I, II, e III, sendo que Strasbourg-II chegou a ostentar o nome de Université Marc Bloch.

3. Paris: Ophrys, 1974.

4. Natural de Metz, estudou também em Estrasburgo, mas a Guerra retardou-lhe o doctorat para 1949. Viria a ensinar longamente em Dijon (1950-1986). De qualquer modo, tornar-se-ia um 
verdadeiro mediador historiográfico entre a Alemanha e a França (foi um notável estudioso de Carlos Magno, da sua memória e da sua lenda, bem como da santidade de reis e rainhas medievais), onde teve o seu papel na divulgação de autores como E.-H. Kantorowicz (1895-1963). Membro correspondente do Institut de France.

5. Membro do Institut de France, natural de Metz, estudou em Besançon e Estrasburgo, e começou por ensinar nos liceus de Sarreguemines, Metz (1932-1939) e Montpellier, e por último na Universidade de Nancy, como chargé d'enseignement (1943). Mas no ano seguinte é preso pela Gestapo e deportado para Struthof e depois para Dachau. Regressa a Nice com o fim da guerra, e em 1948 aí obtém o doctorat d'État (tese: La Ville de Metz aux XIII ${ }^{e}$ et XIV ${ }^{e}$ siècles, 1950). Em Nancy vai ensinar longamente, tornando-se também directeur d'études da École pratique des hautes études (1957-1974).

6. V. infra, bibliografia activa.

7. Cf., respectivamente: L'Occident aux XIV et $X V^{e}$ siècles. Aspects économiques et sociaux. Paris: PUF, 1970 (com reedições) e L'Occident aux XIVee ${ }^{e} X^{e}$ siècles. Les États, 4. ․ ed. Paris: PUF, 1991.

8. V. infra, bibliografia activa.

9. V. infra, bibliografia activa, títulos publicados entre 1971 e 2010.

10. Entre 1970 e 2012, esta Universidade dividiu-se em Nancy-I e Nancy-II. No último ano mencionado, e seguindo uma tendência que é já do século XXI, deu-se a reunificação, que englobou ainda a Universidade Paul Verlaine - Metz e o l'Institut national polytechnique de Lorraine, tudo isto dando origem à nova Universidade da Lorena.

\section{AUTOR}

\section{ARMANDO LUÍS DE CARVALHO HOMEM}

Universidade do Porto, Faculdade de Letras, Departamento de História e de Estudos Políticos e Internacionais; Universidade do Porto, Centro de Estudos de População, Economia e Sociedade; Universidade Autónoma de Lisboa, Centro de Investigação em Ciências Históricas 4099-002 Porto; 1169-023 Lisboa, Portugal. almisch@gmail.com. https://orcid.org/0000-0001-9337-6995 